\title{
Acute encephalitis caused by Schistosoma mansoni
}

\author{
Paulo P. Christo ${ }^{1,2}$, Vírginia A. Zambelli ${ }^{1}$, Frederico F. Amâncio', \\ Marcelo Oliveira', Sílvia H. Carvalho', Renato C. Said ${ }^{1}$
}

Schistosomiasis associated with Schistosoma mansoni is endemic in the Caribbean Islands, the Middle East, South America and Africa and can be imported to any other region of the world by immigration and travel to endemic areas ${ }^{1,2}$. The disease represents a serious public health problem, with an estimated 200 to 300 million people being infected with $S$. mansoni worldwide 2 . In Brazil, 4 to 6 million individuals are estimated to be infected with S. mansoni ${ }^{3}$.

S. mansoni infection is primarily located in the gastrointestinal tract and liver and feeds on blood in the portal and mesenteric vessels, with the central nervous system being less frequently involved. Neuroschistosomiasis is considered to be a rare presentation ${ }^{4,5}$, with the spinal cord being the most commonly affected site ${ }^{4}$. The acute phase of infection can be asymptomatic or oligosymptomatic or may cause signs and symptoms such as fever, macular rash, myalgia, malaise, nonproductive cough, abdominal pain, nausea, vomiting and eosinophilia ${ }^{5}$. Neurological involvement during this phase has been much less frequently reported.

We describe here two cases of patients with acute encephalitis caused by recent infection with S. mansoni.

\section{CASES}

\section{Case 1}

A 13-year-old boy, born and living in Belo Horizonte, Minas Gerais, was admitted with 3-day manifestations of fever, vomiting, mental confusion and prostration. The patient had a 15-day history of diffuse papular-erythematous lesions which showed spontaneous improvement and reported contact with lake water approximately 40 days earlier. There was no report of previous diseases, travels or recent vaccinations and the patient presented no other sign or symptom. Seven children playing in the same lake had shown signs of diarrhea and skin rash. Clinical examination did not demonstrate hepatosplenomegaly or respiratory alterations. Neurological examination revealed that the patient was torpid and confused, with associated conjugate eye deviation to the left, asymmetric tetraparesis, flexion of the upper limbs, global hyperreflexia, and bilateral Babinski sign. There were no signs of neck stiffness. Laboratory exams showed leukocytosis $\left(25,000\right.$ leukocytes $/ \mathrm{mm}^{3}$ and $20 \%$ eosinophils). Ions, anti-nuclear factor (ANF), VDRL, rheumatoid factor, HTLV $1 / 2$, HIV, serology (ELISA) for cysticercosis, liver enzymes and blood culture were normal or negative. Indirect immunofluorescence for the detection of $S$. mansoni in blood was positive (1:80). The parasitological stool exam was also positive for $S$. mansoni (Kato-Katz technique). Analysis of cerebrospinal fluid yielded the following results: 3 cells $/ \mathrm{mm}^{3}$, glucose: $84 \mathrm{mg} / \mathrm{dl}$ and total protein: $95 \mathrm{mg} / \mathrm{dl}$. Direct investigation and culture of cerebrospinal fluid for bacteria, fungi or tuberculosis were negative. A chest X-ray was normal. Magnetic nuclear resonance imaging (MRI) of the brain demonstrated right parietal and left frontal subcortical hypointense lesions without contrast uptake. The prescribed treatment consisted of intravenous corticosteroids for 14 days and a single dose of $50 \mathrm{mg} / \mathrm{kg}$ praziquantel. Initially in view of
Av. Alfredo Balena 189 / sala 1708 30130-100 Belo Horizonte MG - Brasil E-mail: ppc@gold.com.br

Received 25 May 2009

Received in final form 26 November 2009 Accepted 8 December 2009

\section{ENCEFALITE AGUDA CAUSADA PELO SCHISTOSOMA MANSONI}

'Eduardo de Menezes Hospital, FHEMIG, Belo Horizonte MG, Brazil; ${ }^{2}$ MD, PhD, Department of Neurology, Santa Casa de Belo Horizonte, Belo Horizonte MG, Brazil. 
the initial diagnostic suspicion of HSV-1 encephalitis, the patient received empirical treatment with Acyclovir for few days. The patient presented slow and progressive improvement of the neurological signs and symptoms and was discharged 25 days after the onset of symptoms.

\section{Case 2}

A 14-year-old boy living in the metropolitan region of Belo Horizonte was admitted with symptoms of sleepiness and psychomotor agitation. The patient presented a history of headache, vomiting, diarrhea, fever and prostration, which had started 7 days ago, and of urinary incontinence and mental confusion for 3 days. The patient had a papular rash which had developed 30 days earlier after contact with dam water close to his home. There was no report of previous diseases, travels or recent vaccinations and the patient presented no cough, weight loss, drug use or arthralgias. Other individuals who had contact with water from the same dam had reported diarrhea and fever. Neurological examination revealed sleepiness, disorientation in time and space and neck stiffness. The patient presented no focal deficits. Systemic examination demonstrated a palpable and tender. Laboratory exams showed leukocytosis $\left(18,400\right.$ cells $/ \mathrm{mm}^{3}$ and $51 \%$ eosinophils). Creatinine, urea and transaminases were normal. Ions, ANF, VDRL, rheumatoid factor, HTLV 1/2, HIV and ELISA for cysticercosis were normal or negative. Cerebrospinal fluid analysis revealed 6 cells $/ \mathrm{mm}^{3}$, glucose: 55 $\mathrm{mg} / \mathrm{dl}$ and total protein: $48 \mathrm{mg} / \mathrm{dl}$. Cultures and direct investigation of cerebrospinal fluid for tuberculosis, bacteria or fungi were negative. A chest X-ray was normal. An abdominal ultrasound showed hepatosplenomegaly accompanied by discrete enlargement of the splenic vein and no periportal fibrosis. Cranial tomography showed diffuse edema and diffuse contrast extravasation, suggesting a breakdown of the blood-brain barrier. Numerous $S$. mansoni eggs were detected by parasitological stool examination (Kato-Katz). Indirect immunofluorescence for the detection of S. mansoni in blood was positive. The patient was treated with a single dose of $50 \mathrm{mg} / \mathrm{kg}$ praziquantel, which resulted in progressive improvement of signs and symptoms, and was discharged asymptomatic 14 days after admission.

The hospital ethic commission approved this case report and the parents gave informed consent for publication.

\section{DISCUSSION}

There are three clinical syndromes caused by $S$. mansoni. The immediate syndrome is characterized by maculopapular eruption at the site of penetration of the cercaria immediately after infection (cercarial dermatitis) ${ }^{6}$. Acute schistosomiasis or Katayama fever has been described for infections with S. japonicum. This clinical form is rare in patients from endemic areas but highly prevalent in nonimmune western individuals traveling to areas endemic for schistosomiasis ${ }^{5,6}$ and the most common symptoms include fever, headache, shivering, myalgia, anorexia, nausea, vomiting, upper quadrant abdominal pain, diarrhea, and respiratory problems. Hepato- and splenomegaly might be present. Neurological involvement during the acute phase, as observed in the present cases of encephalitis, is rare. The last stage is the chronic phase (chronic schistosomiasis) which results from the immune response of the host to $S$. mansoni eggs and the granulomatous reaction triggered by antigens secreted by the eggs. During this phase, clinical problems involving the gastrointestinal tract and liver are more prevalent since these organs represent the site of maximum egg accumulation ${ }^{5,7}$. The central nervous system may also be affected, but less frequently, with the spinal cord being the most commonly involved site ${ }^{8}$.

Acute infection with schistosoma worms (acute schistosomiasis) is a systemic hypersensitivity reaction to migration of the young worm which occurs within a few weeks or months after primary infection ${ }^{6,9,10}$. Encephalitis or acute encephalomyelitis accompanying this syndrome has been described less frequently but may occur during this phase ${ }^{4}$. Neurological symptoms include confusion, focal and generalized seizures, visual alterations, and papilledema. Signs of encephalopathy such as hemiplegia and Babinski sign or evidence of myelopathy characterized by lower limb weakness, paresthesia, sensory loss and sphincter disorders may also be observed ${ }^{6}$. The first patient presented manifestations of focal encephalitis characterized by conjugate eye deviation and motor deficits, with MRI showing hypointense areas without contrast uptake. The second patient presented manifestations of meningoencephalitis accompanied by changes in consciousness and neck stiffness. These manifestations have been attributed to a humoral-like immune response to the adult worm or egg antigens, which is characterized by an increase of serum immunoglobulins and the deposition of immune complexes ${ }^{1}$.

Acute encephalitis is a neurological emergency and early treatment based on clinical diagnostic probability is imperative. Positive epidemiology demonstrated by contact with lake water and other individuals with systemic symptoms close to the patient, the observation of a rash and subsequent demonstration of eosinophilia were findings that helped in the establishment of the diagnosis for the present patients.

After contact with contaminated waters, cercariae penetrate the skin, migrate to the liver in the bloodstream via the lung and transform into young worms. Maturation occurs in the portal vein within 4-6 weeks and the young worms migrate to the mesenterium where 
the cycle starts again ${ }^{5}$. The onset of neurological symptoms coincides with the phase of oviposition, when eggs are detected in the parasitological stool exam. Microscopic analysis of stool samples remains the gold standard for the diagnosis of schistosomiasis ${ }^{11}$. Antibody-based tests are very sensitive but do not distinguish between active and past infection and cross-reactions with other helminths might occur ${ }^{12}$.

The present patients were living in a metropolitan region. Although schistosomiasis is mainly a rural problem, urban foci might be found in many endemic areas ${ }^{13}$. The peak of acute infection is generally observed at about 8-15 years of age and declines with age, probably because of acquired and innate immunity ${ }^{14}$. Acute infection is rare in populations chronically exposed to $S$. mansoni due to underdiagnosis or to intrauterine sensitization ${ }^{15}$. Individuals living in endemic areas for long periods of time acquire some type of immune resistance ${ }^{14}$.

The possibility of schistosoma etiology in cases of acute encephalitis should always be considered in endemic areas for schistosomiais or in patients with positive epidemiological history, including persons traveling to rural areas due to the proliferation of rural tourism among inhabitants of large Brazilian cities.

\section{REFERENCES}

1. Pitella JE. Neuroschistosomiasis. Brain Pathol 1997;7:649-662.

2. World health Organization. Schistosomiasis. Geneva, World Health Organization 1996;1-4.

3. Drummond SC, Silva LC, Amaral RS, Sousa-Pereira SR, Antunes CM, Lambertucci JR. Morbidity of Schistosomiasis mansoni in the state of Minas Gerais,Brazil. Mem Inst Oswaldo Cruz 2006;101:37-44.

4. Ferrari TCA. Involvement of central nervous system in the schistosomiasis. Mem Inst Oswaldo Cruz 2004:99:59-62.

5. Ross AG, Bartley PB, Sleigh AC, et al. Schistosomiasis. N Engl J Med 2002;346: $1212-1220$

6. Lambertucci JR. Acute schistosomiasis: clinical, diagnostic and therapeutic features. Rev Inst Med Trop Sao Paulo 1993;35:399-404.

7. Betting LE, Pirani Jr C, Queiroz LS, et al. Seizures and Cerebral schistosomiasis. Arch Neurol 2005;62:1008-1010.

8. Ministério da Saúde - Secretaria de Vigilância em Saúde Guia de Vigilância epidemiológica e controle da mielorradiculopaia esquistossomótica série A. Normas e Manuais Técnicos, Brasília, Distrito Federal, 2006.

9. Bottieau E, Clerinx J, de Vega MR, et al. Imported Katayama fever: clinical and biological features at presentation and during treatment. J Infect 2006 52:339-345.

10. Rocha MO, Greco DB, Pedroso ER, et al. Secondary cutaneous manifestations of acute schistosomiasis mansoni. Ann Trop Med Parasitol 1995;89: 425-430.

11. Feldmeirer $\mathrm{H}$, Poggensee $\mathrm{G}$. Diagnostic techiniques in schistosomiasis control: a review. Acta Trop 1993;52:205-220.

12. Rabello A. Diagnosing schistosomiasis. Mem Inst Oswaldo Cruz. 1997;92: 669-676.

13. Mott KE, Desjeux P, Moncayo A, Ranque P, de Raadt P. Parasitic diseases and urban development. Bull World Health Organ 1990;68:691-698.

14. Gryseels B. Uncertainties in the epidemiology and control of schistosomiasis. Am J Trop Med Hyg 1996;55:103-108.

15. Gryssels B, Polman K, Clerinx J, Kestens L. Human schistosomiasis. Lancet 2006;368:1106-1118. 